\title{
659 T CELL RECEPTOR EXCHANGE BY ZYGOTE ENGINEERING RESULTS IN PHYSIOLOGICAL T CELL RESPONSES FOR THERAPEUTIC USE IN PANCREATIC DUCTAL ADENOCARCINOMA
}

Meagan Rollins*, Jackson Raynor, Ebony Miller, Ellen Spartz, Walker Lahr, Adam Burrack, Yun You, Branden Morarity, Beau Webber, Ingunn Stromnes. University of Minnesota, Minneapolis, MN, United States

Background Pancreatic ductal adenocarcinoma (PDA) is a lethal malignancy characterized by a highly suppressive tumor microenvironment. Despite this, engineered $\mathrm{T}$ cell therapy has promise for effectively targeting PDA. To identify the underlying mechanisms of antigen-specific engineered $\mathrm{T}$ cell immunosuppression in PDA, we create novel TCR knock-in mouse models for a robust and standardized source of naïve mesothelin (Msln)-specific T cells.

Methods Specifically, we integrate two murine mesothelin-specific TCRs into the physiologic Trac locus in primary murine $\mathrm{T}$ cells and zygotes using CRISPR/Cas9 and rAAV expressing the TCR DNA. Simultaneously using CRISPR/Cas9, Msln was disrupted to circumvent $\mathrm{T}$ cell tolerance.

Results This strategy resulted in the rapid generation of homozygous TCR Trac knock-in mice and with homozygous null mutations in Msln. In these TCR-exchanged (TRex) mice, most $\mathrm{T}$ cells expressed the 1045 (high affinity) or 7431 (low affinity) as determined by tetramer staining. TRex $\mathrm{T}$ cells exhibit a naïve phenotype and rapidly differentiate into effector $\mathrm{T}$ cells upon antigenic stimulation. While the high affinity 1045 TCR elicits function in CD4 T cells, the lower affinity $7431 \mathrm{~T}$ cells exhibit a higher functional avidity and less TCR downregulation when antigen is limiting. Historical TCR transgenic $\mathrm{T}$ cells, in which the TCR is randomly integrated into the genome, exhibit increased PD1, CD25, and CD69, decreased functionality, and a bias to CD25-Foxp3+ Treg as compared to T cells from TRex mice. Further, TCR Trac integration in primary $\mathrm{T}$ cells retain superior function following repetitive antigenic stimulations retrovirally transduced $\mathrm{T}$ cells. Adoptive transfer of 1045 TRex T cells significantly prolongs survival of mice bearing autochthonous PDA. When combined with a vaccine, 1045 TRex $\mathrm{T}$ cells cause involution of the fibroinflammatory tumor stroma.

Conclusions In sum, we rapidly generate mice that physiologically express the desired TCR, circumventing the shortcomings of standard $\mathrm{T}$ cell engineering strategies and TCR transgenic models.

Ethics Approval University of Minnesota Institutional Animal Care and Use Committee approved all animal studies to Dr. Ingunn Stromnes (2005-38115A.) Generation of TCR knockin (KI) animals was performed in the Mouse Genetic Laboratory at the University of Minnesota.

http://dx.doi.org/10.1136/jitc-2021-SITC2021.659 